\title{
Effect on macrophage proliferation of a novel polysaccharide from Lactarius deliciosus (L. ex Fr.) Gray
}

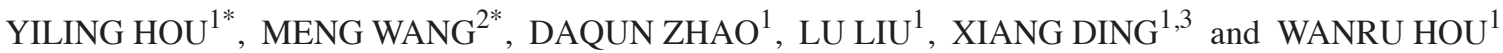 \\ ${ }^{1}$ Key Laboratory of Southwest China Wildlife Resources Conservation, College of Life Sciences, \\ China West Normal University, Nanchong, Sichuan 637009; ${ }^{2}$ Department of Emergency Medicine, \\ Children's Hospital Affiliated to Zhengzhou University, Zhengzhou, Henan 450000; \\ ${ }^{3}$ College of Environmental Science and Engineering, China West Normal University, Nanchong, Sichuan 637009, P.R. China
}

Received April 9, 2016; Accepted December 4, 2018

DOI: $10.3892 / \mathrm{ol} .2018 .9879$

\begin{abstract}
The fundamental mechanisms underlying the preventional and therapeutic effects of polysaccharides from fungi, including the immunostimulatory, antiviral and antitumor effects, are considered to occur through the modulation and stimulation of the macrophage and complement system. LDG-A, a novel polysaccharide from Lactarius deliciosus (L. ex Fr.) Gray exhibits marked antitumor activities in vivo. However, the underlying molecular mechanism of the antitumor activities of LDG-A remains unclear. In the present study, cell cycle analysis was performed in macrophages and B cells, and the transcriptomes of macrophages in the control group and LDG-A group were sequenced using Illumina sequencing technology to analyze the differentially expressed genes (DEGs), and elucidate the molecular mechanisms underlying the immunomodulatory and antitumor activities of LDG-A. The cell cycle analysis results indicated that LDG-A was able to promote the proliferation of $\mathrm{B}$ cells by promoting cell cycle progression in $\mathrm{S}$ phase and $\mathrm{G}_{2} / \mathrm{M}$ phase and eliminating cell cycle arrest in $\mathrm{G}_{0} / \mathrm{G}_{1}$, and promote the proliferation of macrophages by promoting cell cycle progression in $G_{0} / G_{1}$ phase and eliminating cell cycle arrest in $\mathrm{G}_{2} / \mathrm{M}$ phase. Of the total number of genes $(8,140), \sim 77.00 \%$ were expressed [reads per kilobase per million reads $(\mathrm{RPKM}) \geq 1$ ] and 1,352 genes were highly expressed (RPKM $>60)$ in the LDG-A group. Of 775 unigenes which were identified as DEGs, 469 were downregulated and 306 genes were upregulated. A protein chip method was also used to determine the cytokines secreted by macrophages. Kyoto Encyclopedia of Genes and Genomes
\end{abstract}

Correspondence to: Dr Xiang Ding, College of Environmental Science and Engineering, China West Normal University, 1 Shida Road, Nanchong, Sichuan 637009, P.R. China

E-mail: biostart8083@126.com

*Contributed equally

Key words: Lactarius deliciosus (L. ex Fr.) Gray, polysaccharide, proliferation effect, macrophage, RNA sequencing, protein chip pathway enrichment analysis and GO enrichment analysis indicated that the Janus kinase/signal transducer and activator of transcription, mitogen-activated protein kinase, chemokine, vascular endothelial growth factor and transforming growth factor $\beta$ signaling pathways are markedly enriched for DEGs.

\section{Introduction}

In recent years, polysaccharides from fungi have attracted increasing attention for the prevention and treatment of cancer because of their efficiency in tumor inhibition and low toxicity $(1,2)$. Immunomodulatory and antitumor properties are usually associated with the ability to activate the immune and complement systems (3). The function of immune system is to recognize and eliminate antigenic foreign bodies, co-ordinating with other systems of the body, and maintaining the stability of the internal environment and physiological balance of the body. B lymphocytes, also called B cells, are a pluripotent stem cell derived from bone marrow $(4,5)$. Macrophages participate in non-specific defense (congenital immunity) and specific defense (cellular immunity) in vertebrates. Their main function is to phagocytose cell debris and pathogens in the form of fixed or free cells (i.e., phagocytosis and digestion), and activate lymphocytes or other immune cells to react to pathogens (6). B cells and macrophages can initiate natural immune responses and then act as effector cells which help to manage the immune responses, such as fighting an infection, angiogenesis and inflammation $(7,8)$.

Polysaccharides are ubiquitous substances in organisms. They are natural macromolecule polymers linked by aldose or ketose through glycosidic bonds. They are important biological macromolecules in organisms and are basic substances to maintain normal operation of cellular activities (9). A number of polysaccharides (including polysaccharides from plants, animals and fungi) regulate or promote immune function. Their primary functions are to enhance the activities of the reticuloendothelial system, macrophages, natural killer cells, killer T cells, lymphokine-activated killer cells and other cytokines, and to promote antibody, complement production, protein synthesis, etc. (10). The biological activity of polysaccharides depends on the process of recognition and interaction between polysaccharides and their receptors. 
When polysaccharides interact with receptors, specific oligosaccharide fragments often bind to receptors, recognizing the activation of polysaccharide receptors by ligands to initiate intracellular signal transduction pathways and thus produce effectual functions (11-13).

Our group recently isolated a novel polysaccharide from Lactarius deliciosus (L. ex Fr.) Gray named LDG-A, which has a backbone of 1,6-disubstituted- $\alpha$-L-mannopyranose which is branched at $\mathrm{O}-2$, with branches mainly composed of $\alpha \rightarrow 3$ - $\alpha$-D-xylopyranose residues (14). LDG-A also exhibits significant antitumor activities in vivo, and activates lymphocytes and macrophages in vitro (15). However, the molecular mechanism underlying antitumor and immunomodulatory activity of LDG-A remain unclear. In the present study, cell cycle analysis of macrophages and B cells was performed and the transcriptomes of macrophages of the control group and LDG-A group were sequenced using Illumina sequencing technology. Protein chip technology was also used to determine the cytokines secreted by macrophages. The aim of the present study was to determine the molecular mechanism underlying the effect of polysaccharide LDG-A in antitumor and immune regulation.

\section{Materials and methods}

Materials. RPMI-1640 medium, D-Hanks solution, dimethylsulfoxide and fetal bovine serum (FBS) were purchased from Gibco; Thermo Fisher Scientific, Inc. (Waltham, MA, USA), The Cell Counting kit-8 (CCK-8) reagent was purchased from Dojindo Molecular Technologies, Inc. (Tokyo, Japan). Lipopolysaccharide (LPS), streptomycin and penicillin G were purchased from Sigma; Merck KGaA (Darmstadt, Germany). The RAW264.7 cell line and B cell line (Raji) were purchased from the Institute of Biochemistry and Cell Biology (Shanghai Academy of Life Sciences, Chinese Academy of Sciences). LDG-A was prepared in our laboratory as described previously (14). All other chemicals and solvents used were of analytical grade.

Cell lines and reagents. D-Hanks solution was used for cell digestion and passage. The dimethylsulfoxide was used for cell cryopreservation. The CCK-8 reagent was used for cell activity detection. The RAW264.7 cell line and B cell line (Raji) were cultured at $37^{\circ} \mathrm{C}$ in a humidified atmosphere containing $5 \% \mathrm{CO}_{2}$ in RPMI-1640 medium containing $1 \%$ penicillin $\mathrm{G}$ $(100 \mathrm{IU} / \mathrm{ml}), 10 \% \mathrm{FBS}$ and streptomycin $(100 \mathrm{mg} / \mathrm{l})$.

RAW264.7 cell and B cell cycle analysis by flow cytometry. Cells were stained using a cell apoptosis detection kit (cat. no. C1052; Beyotime Institute of Biotechnology, Haimen, China). Following staining of the cells with propidium iodide (PI), the effect of LDG-A on the cell cycle distribution was determined using flow cytometry. RAW264.7 cells and B cells $\left(5 \times 10^{5}\right.$ cells/well) were cultured and exposed to LDG-A $(1,5$ or $10 \mu \mathrm{g} / \mathrm{ml})$ for $24 \mathrm{~h}$ and then washed with PBS twice and fixed in ice-cold $70 \%$ ethanol for $4 \mathrm{~h}$ at $4^{\circ} \mathrm{C}$. Following an additional wash in PBS, the cells were resuspended in staining buffer $(0.5 \mathrm{ml})$ containing $25 \mu \mathrm{l}$ PI and $10 \mu 1$ RNase, then incubated at $37^{\circ} \mathrm{C}$ in the dark for $30 \mathrm{~min}$. The DNA content of the cells was determined using a flow cytometer (BD Accuri C6; San Jose, CA, USA), and the cell population was calculated in each phase using the ModFit LT program (version 2.0, Verity Software House Inc., Topsham, ME, US). Each experiment was performed three times.

RNA extraction, library preparation and high-throughput sequencing. The LDG-A concentration used to analyze the transcriptome of RAW264.7 cells was $15 \mu \mathrm{g} / \mathrm{ml}$. Total RNA was extracted using TRIzol ${ }^{\circledast}$ reagent (Invitrogen; Thermo Fisher Scientific, Inc.), and the RNA quality and purity were determined using $1 \%$ agarose gels (16). RNA purity was analyzed at a wavelength of $260 \mathrm{~nm}$ using a NanoPhotometer spectrophotometer (Implen, Inc., Westlake Village, CA, USA). The transcriptome libraries were generated using the Illumina TruSeq ${ }^{\mathrm{TM}}$ RNA Sample Preparation kit (Illumina, Inc., San Diego, CA, USA). The libraries were sequenced on an Illumina HiSeq 2000 platform.

Transcriptome data analysis. The raw data in FASTQ format were processed using in-house Perl scripts to remove low-quality reads, including poly- $\mathrm{N}$ stretches (partially unsequenced regions) and adapter sequences. The high-quality clean sequences were used for the downstream analyses.

Differential expression and quantification analysis of the transcripts. An edgeR program package of Bioconductor (version 3.8; Affymetrix; Thermo Fisher Scientific, Inc.) was used for each sequenced library through one scaling normalized factor to adjust the read counts prior to the differential gene expression analysis and the DEGSeq $\mathrm{R}$ package was used to identify the DEGs between the two cell groups. Values of $\log _{2}$ ratiol $\geq 1$ and FDR $\leq 0.001$ were set as the thresholds for significant differential expression. The transcript level expression was quantified using the reads per kilobase per million reads (RPKM) method (17) and the number of reads mapped to each transcript was determined using HTSeq (version 0.5.3, Affymetrix; Thermo Fisher Scientific, Inc.). The RPKM value was calculated on the basis of the sequencing depth, mapped transcript fragments and transcript length. The read counts with one scaling normalized factor were completed using the edgeR Bioconductor package prior to the differential gene expression analysis. The threshold of statistically significant differential expression was a P-value of 0.05 and a $\log _{2}$ fold change of \pm 1 . A l $\log _{2}$ fold changel $>5$ was used to identify differentially expressed genes (DEGs).

Protein chip assay. RAW264.7 cells $\left(5 \times 10^{5}\right.$ cells/well) were cultured and exposed to LDG-A $(15 \mu \mathrm{g} / \mathrm{ml})$ for $24 \mathrm{~h}$. Cells were collected and centrifuged at $300 \mathrm{xg}$ at $4^{\circ} \mathrm{C}$ for $2 \mathrm{~min}$. The supernatant was used to perform the protein chip assay. The mouse cytokine array reagent kit (cat. no. QAM-CAA-4000; RayBiotech Life, Norcross, GA, USA) was used according to the manufacturer's protocol.

Differential expression and quantification analysis of the cytokines. The experimental data were extracted following the protein chip assay using the microarray analysis software ArrayVision (version 7.0; Affymetrix; Thermo Fisher Scientific Inc.). The quantitative data were analyzed using Quantibody Q-Analyzer software (version R; Affymetrix; Thermo Fisher Scientific Inc.). 


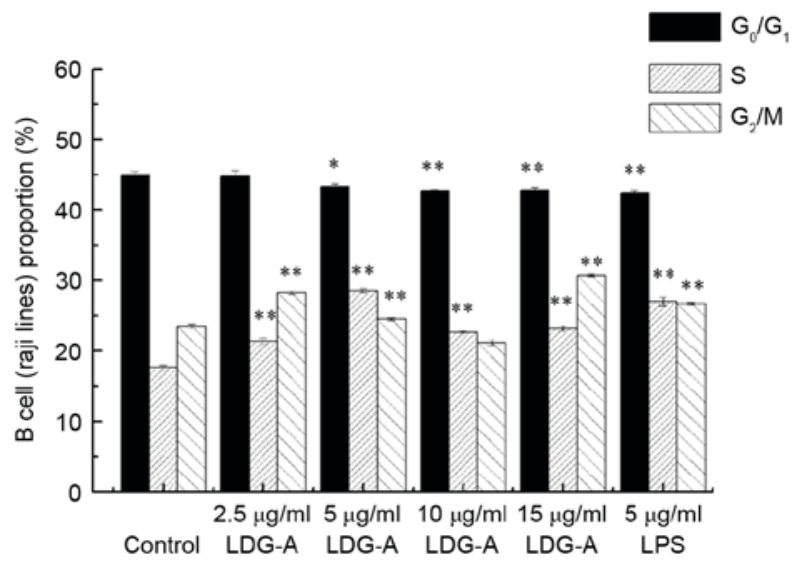

Figure 1. Effects of LDG-A on the cell cycle distribution of B cells. Results are presented as the mean \pm standard deviation $(n=3)$. " $\mathrm{P}<0.05$, ${ }^{* *} \mathrm{P}<0.01$ vs. control group. LPS, lipopolysaccharide.

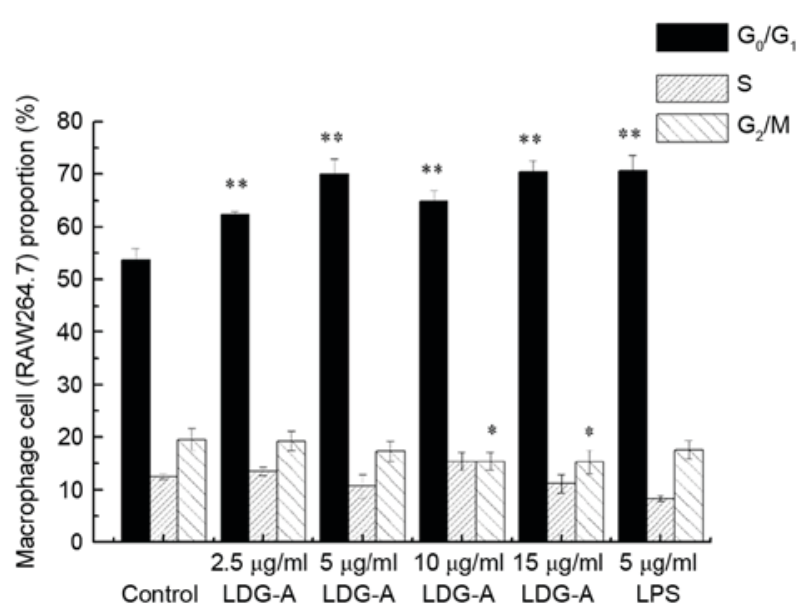

Figure 2. Effects of LDG-A on the cell cycle distribution of RAW264.7 cells. Results are presented as the mean \pm standard deviation $(n=3)$. ${ }^{*} \mathrm{P}<0.05$, ${ }^{* *} \mathrm{P}<0.01$ vs. control group. LPS, lipopolysaccharide.

Gene Ontology (GO) annotation and GO/Kyoto Encyclopedia of Genes and Genomes (KEGG) enrichment analyses. BLASTX and InterProScan against the National Center for Biotechnology Information database was used to annotate the protein functions of all genes. The resulting InterPro annotations and BLASTX results were then converted into GO annotations. All GO terms were mapped onto the GO Slim categories. The statistical significance of the functional GO Slim enrichment was determined using Fisher's exact test within Blast2GO [false discovery rate (FDR) <0.05]. The significantly enriched KEGG pathways were identified using the hypergeometric test and the Benjamini-Hochberg FDR correction with KEGG Orthology-Based Annotation System (KOBAS; version 2.0; Affymetrix; Thermo Fisher Scientific, Inc.).

Statistical analysis. All results are presented as the mean \pm standard deviation of three replicates. Statistical analyses were performed using Student's t-test and one-way analysis of variance followed by Student-Newman-Keuls test with SPSS (version 10.0; SPSS, Inc., Chicago, IL, USA). $\mathrm{P}<0.05$ was considered to indicate a statistically significant difference.

\section{Results}

Effect of LDG-A on the cell cycle distribution of B cells. Cell cycle analysis of B cells was performed using flow cytometry to investigate the effects of LDG-A on cell cycle progression. Fig. 1 presents the results of the effect of LDG-A on the cell cycle phase distribution $\left(G_{0} / G_{1}, S\right.$ and $\left.G_{2} / M\right)$ of B cells with PI staining using flow cytometry. The treatment of B cells with LDG-A for $24 \mathrm{~h}$ at 2.5, 5, 10 and $15 \mu \mathrm{g} / \mathrm{ml}$ induced a concomitant decrease in the percentage of cells in $\mathrm{G}_{0} / \mathrm{G}_{1}$ phase from 44.9 (control group) to 45.0, $43.3(\mathrm{P}<0.05), 42.7(\mathrm{P}<0.01)$ and $42.8 \%(\mathrm{P}<0.01)$, respectively, with a concentration-dependent and significant increase in the $\mathrm{G}_{2} / \mathrm{M}$-phase population from 23.5 (control group) to $30.7 \%$ of the LDG-A $(15 \mu \mathrm{g} / \mathrm{ml})$ group $(\mathrm{P}<0.01)$. LDG-A, at concentrations of $2.5,5,10$ and $15 \mu \mathrm{g} / \mathrm{ml}$, also induced a significant increase in the $\mathrm{S}$-phase population, from $17.6 \%$ (control group) to $21.2 \%(\mathrm{P}<0.01), 28.6 \%$ $(\mathrm{P}<0.01), 22.7 \%(\mathrm{P}<0.01)$ and $23.2 \%(\mathrm{P}<0.01)$, respectively. In the positive control group, $5 \mu \mathrm{g} / \mathrm{ml}$ LPS could promote B cell proliferation by inhibiting $\mathrm{G}_{0} / \mathrm{G}_{1}$ phase and promoting $\mathrm{S}$ phase and $\mathrm{G}_{2} / \mathrm{M}$ phases. These results suggested that LDG-A could promote the proliferation of $\mathrm{B}$ cells by promoting cell cycle progression in $\mathrm{S}$ phase and $\mathrm{G}_{2} / \mathrm{M}$ phases, and eliminating cell cycle arrest in $G_{0} / G_{1}$ phase, which may induce cell division.

Effect of LDG-A on the cell cycle distribution of RAW264.7 cells. The primary function of macrophages is to phagocytose cell debris and pathogens in the form of fixed cells or free cells (phagocytosis and digestion), and activate lymphocytes or other immune cells to react to pathogens. Fig. 2 presents the results of the effect of LDG-A on the cell cycle phase $\left(G_{0} / G_{1}, S\right.$ and $\mathrm{G}_{2} / \mathrm{M}$ ) distribution of macrophages RAW264.7 cells with PI staining using flow cytometry. The treatment of RAW264.7 cells with LDG-A at 2.5, 5, 10 and $15 \mu \mathrm{g} / \mathrm{ml}$ for $24 \mathrm{~h}$ induced a concomitant increase in the proportion of cells in $\mathrm{G}_{0} / \mathrm{G}_{1}$ phase from 53.8 for the control group to $62.4(\mathrm{P}<0.01), 70.0(\mathrm{P}<0.01)$, $64.8(\mathrm{P}<0.01)$ and $70.5 \%(\mathrm{P}<0.01)$, respectively, with a concentration-dependent decrease in the $\mathrm{G}_{2} / \mathrm{M}$ phase population from 19.9 for the control group to $19.2,17.0,15.4(\mathrm{P}<0.05)$ and $15.1 \%$ $(\mathrm{P}<0.05)$, respectively. In the positive control group, $5 \mu \mathrm{g} / \mathrm{ml}$ LPS could promote macrophages RAW264.7 cell proliferation by inhibiting $S$ phase and $G_{2} / M$ phases and promoting $G_{0} / G_{1}$ phases. These results suggested that LDG-A could promote the proliferation of macrophage cells by promoting cell cycle progression in $G_{0} / G_{1}$ phase and eliminating cell cycle arrest in $\mathrm{G}_{2} / \mathrm{M}$ phases, which may also induce cell division.

Transcriptome sequencing and de novo assembly. High-throughput sequencing technology was used to investigate the differences in the RAW264.7 cell transcriptomes between the control and the LDG-A group. The respective total RNA from the control group and LDG-A group was used to construct the two cDNA libraries. An Illumina HiSeq 2000 platform was used to sequence the prepared libraries. Following removal of the low-quality reads and adapter sequences, a total of 11,620,079 and 12,498,414 bp paired-end reads were obtained for the LDG-A and control groups, respectively, which corresponded to a total size of 11.6 and $12.5 \mathrm{Gbp}$, respectively (Table I). The clean reads were mapped onto the RAW264.7 cell reference genome. The proportion of total 
Table I. Summary of the mapping results (mapping to reference genome).

\begin{tabular}{lcccccccc}
\hline Sample & Total reads & Total base pairs & $\begin{array}{c}\text { Total mapped } \\
\text { reads }\end{array}$ & $\begin{array}{c}\text { Perfect } \\
\text { matches }\end{array}$ & $\begin{array}{c}\leq 2 \mathrm{bp} \\
\text { mismatches }\end{array}$ & $\begin{array}{c}\text { Unique } \\
\text { matches }\end{array}$ & $\begin{array}{c}\text { Multi-position } \\
\text { matches }\end{array}$ & $\begin{array}{c}\text { Total unmapped } \\
\text { reads }\end{array}$ \\
\hline Control & $12,498,414$ & $612,422,286$ & $5,129,004$ & 985,094 & $4,143,910$ & $3,166,326$ & $1,962,678$ & $7,369,410$ \\
& $(100.00 \%)$ & $(100.00 \%)$ & $(41.04 \%)$ & $(7.88 \%)$ & $(33.16 \%)$ & $(25.33 \%)$ & $(15.70 \%)$ & $(58.96 \%)$ \\
LDG-A & $11,620,079$ & $569,383,871$ & $5,054,912$ & 930,977 & $4,123,935$ & $3,032,909$ & $2,022,003$ & $6,565,167$ \\
& $(100.00 \%)$ & $(100.00 \%)$ & $(43.50 \%)$ & $(8.01 \%)$ & $(35.49 \%)$ & $(26.10 \%)$ & $(17.40 \%)$ & $(56.50 \%)$ \\
\hline
\end{tabular}

Table II. Quantification of gene expression in the LDG-A group (RPKM $>10,000)$.

\begin{tabular}{|c|c|c|c|c|c|c|c|}
\hline $\begin{array}{l}\text { NCBI gene } \\
\text { identifier }\end{array}$ & $\begin{array}{l}\text { Number of } \\
\text { unique reads }\end{array}$ & Length, bp & Coverage, $\%$ & RPKM & Symbol & Description & $\begin{array}{l}\text { KEGG } \\
\text { orthology }\end{array}$ \\
\hline 25319 & 240,012 & 828 & 99.88 & $78,249.08$ & Fth1 & Ferritin, heavy polypeptide 1 & K00522 \\
\hline 171361 & 160,104 & 1,737 & 98.22 & $24,881.64$ & Eef1a1 & $\begin{array}{l}\text { Eukaryotic translation } \\
\text { elongation factor } 1 \alpha 1\end{array}$ & K03231 \\
\hline 81776 & 25,825 & 466 & 93.56 & $14,959.98$ & Rps24 & Ribosomal protein S24 & K02974 \\
\hline 29282 & 28,702 & 518 & 91.89 & $14,957.50$ & $\mathrm{Rpl} 23$ & Ribosomal protein L23 & K02894 \\
\hline 94266 & 14,657 & 366 & 95.08 & $10,810.37$ & Rps27 & Ribosomal protein S27 & K02978 \\
\hline 116646 & 30,486 & 794 & 95.47 & $10,364.70$ & Tpt1 & $\begin{array}{l}\text { Tumor protein, translationally } \\
\text { controlled } 1\end{array}$ & K02894 \\
\hline 29304 & 30,550 & 801 & 81.52 & $10,295.69$ & Rps6 & Ribosomal protein S6 & K02991 \\
\hline
\end{tabular}

NCBI, National Center for Biotechnology Information; RPKM, reads per kilobase per million reads; KEGG, Kyoto Encyclopedia for Genes and Genomes.

reads that mapped onto the genome in the two RAW264.7 cell transcriptome libraries ranged between 41.04 and $43.50 \%$. The sequencing saturation analysis indicated that the number of genes detected by the library was saturated. The 5'-3' sequence preference statistical analysis indicated that the bias at the two ends was limited and the sequencing was primarily focused on the gene body region. The distribution of gene coverage (presented in Fig. 3A) provides a good basis for the follow-up analysis.

Transcriptome profiles of the two RAW264.7 cell groups. The abundance of all genes was normalized and calculated by the RPKM method using uniquely mapped reads. The distribution of the expression levels for the two groups of all the genes was similar. Genes with RPKMs in the interval 0-1 were considered to be expressed at low levels or not expressed at all, and genes with RPKM >60 were considered to be expressed at a high level. The results indicated that in the LDG-A group, $\sim 77.00 \%$ of the total number of genes $(8,140)$ were expressed (RPKM $\geq 1$ ) and $>1,352$ genes were highly expressed (RPKM >60), whereas in the control group, $\sim 81.77 \%$ of the total number of genes $(8,208)$ were expressed $(\mathrm{RPKM} \geq 1)$ and $>1,333$ genes were highly expressed (RPKM $>60)$.

The results also indicated that, in the LDG-A group, there were seven genes (Fth1, Eef1a1, Rps24, Rpl23, Rps27, Tpt1 and Rps6) that were highly expressed (RPKM $>10,000$; Table II). It is worthy of note that the RPKM of the Fth1 gene was 78,249 in the LDG-A group, but only 16,044 in the control group.
Identification of DEGs between the control and LDG-A groups. The edgeR program with one scaling normalized factor was used to adjust the reads. On the basis of the $\log _{10}$-transformed RPKMs of the two cell groups, hierarchical clustering for all the DEGs was performed to identify the gene expression patterns (Fig. 3B). In total, 775 unigenes were identified as the DEGs, and 469 genes were downregulated, whereas 306 genes were upregulated (Fig. 3B). The numbers of DEGs in the control group compared with in the LDG-A group were 27 for transcripts detected with $\log _{2}$ fold changel $>5$ and 201 for transcripts detected with $\log _{2}$ fold changel $>2$. In total, 12 genes were upregulated among the DEGs within the $\log _{2}$ fold changel $>5$ threshold, including Lcn2, Mir22, Mreg, Nos2 and Ap5s1, whereas 15 genes were downregulated, including C1qc, Gpr34, RT1-Db2, Sult1a1 and Kmo, which were the top five upregulated and downregulated genes (Table III).

GO and KEGG enrichment analyses of the DEGs. The functional classifications of the annotated unigenes were confirmed and the transcripts with known proteins were classified using GO analyses. A total of 39,271 genes were converted into generic GO Slim terms and annotated using GO terms. Fisher's exact test in Blast2GO was used to perform the GO enrichment analysis and to analyze the gene functions of the DEGs. In the category of cellular components, 71.5 and $71.2 \%$ of the unigenes were classified as intracellular (GO 0005622) and intracellular part (GO 0044424), respectively. Under the molecular functions, the majority of the GO terms were classified as binding 
Table III. Differentially expressed genes: Upregulated and downregulated ( $\log _{2}$ fold changel $\left.>8\right)$.

\begin{tabular}{|c|c|c|c|c|c|}
\hline $\begin{array}{l}\text { NCBI gene } \\
\text { identifier }\end{array}$ & $\begin{array}{l}\text { Gene length, } \\
\text { bp }\end{array}$ & $\begin{array}{c}\log _{2} \text { ratio } \\
(\mathrm{LDG}-\mathrm{A} / \text { control })\end{array}$ & Symbol & Description & KEGG orthology \\
\hline 170496 & 876 & 11.88811 & $\operatorname{Lcn} 2$ & Lipocalin 2 & K01830, K03999 \\
\hline 100314001 & 95 & 11.85097 & Mir22 & MicroRNA 22 & - \\
\hline 501162 & 645 & 9.47469 & Mreg & Melanoregulin & - \\
\hline 24599 & 3,793 & 8.44597 & Nos2 & Nitric oxide synthase 2 & K13241 \\
\hline 499893 & 1,130 & 8.38563 & Ap5s1 & $\begin{array}{l}\text { Adaptor-related protein } \\
\text { complex } 5, \sigma 1 \text { subunit }\end{array}$ & - \\
\hline 362634 & 1,060 & -9.05405 & $\mathrm{C} 1 \mathrm{qc}$ & $\begin{array}{l}\text { Complement component } 1 \text {, } \\
\text { q subcomponent, } \mathrm{C} \text { chain }\end{array}$ & K03988 \\
\hline 554353 & 1,299 & -8.76072 & Gpr34 & G-protein-coupled receptor 34 & K08383 \\
\hline 24981 & 1,134 & -8.73430 & RT1-Db2 & RT1 class II, locus Db2 & K06752 \\
\hline 83783 & 1,227 & -8.62059 & Sult1a1 & $\begin{array}{l}\text { Sulfotransferase family, cytosolic, } \\
1 \mathrm{~A} \text {, phenol-preferring, member } 1\end{array}$ & K01014 \\
\hline 59113 & 1,733 & -8.47609 & Kmo & $\begin{array}{l}\text { Kynurenine 3-mono-oxygenase } \\
\text { (kynurenine 3-hydroxylase) }\end{array}$ & K00486 \\
\hline
\end{tabular}

NCBI, National Center for Biotechnology Information; KEGG, Kyoto Encyclopedia of Genes and Genomes.

A

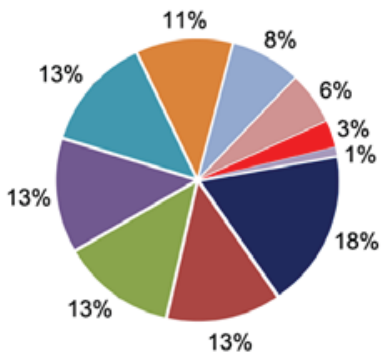

Distribution of genes' coverage (LDG-A)

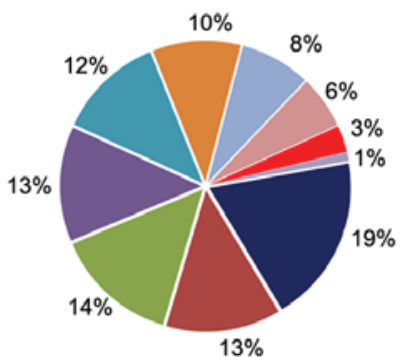

- $90 \%-100 \%(114)$

- $80 \%-90 \%(350)$

$70 \%-80 \%(562)$

- $60 \%-70 \%(816)$

$50 \%-60 \%(1048)$

- $40 \%-50 \%$ (1255)

$=30 \%-40 \%(1330)$

= $20 \%-30 \%$ (1394)

- $10 \%-20 \%(1332)$

= $0 \%-10 \%(1893)$
B

Gene expression level of control vs LDG-A

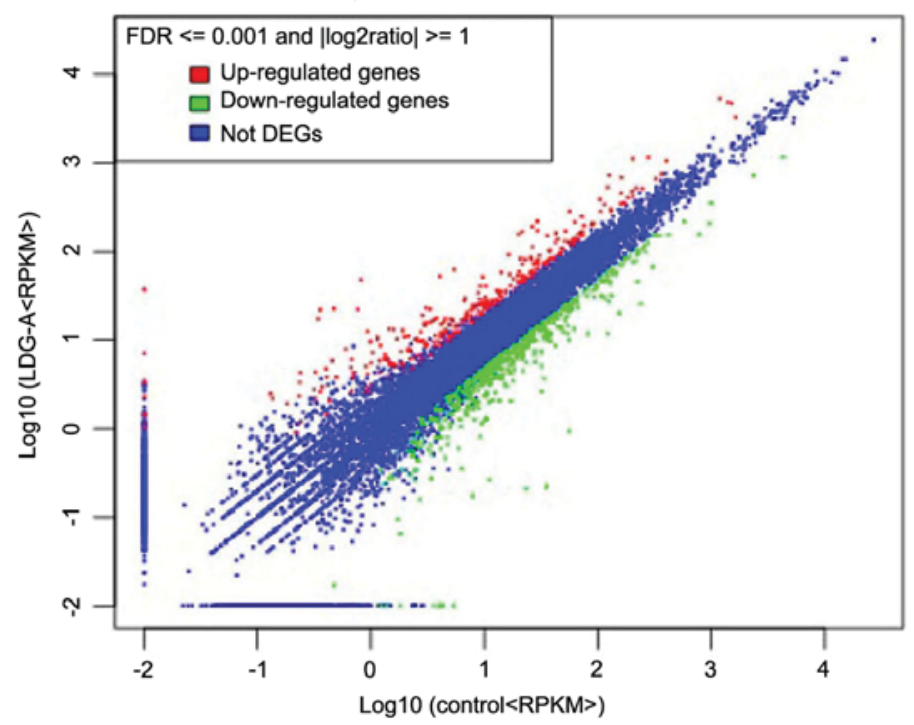

Figure 3. (A) Distribution of gene coverage. (B) Differential expression of genes. FDR, false discovery rate; DEG, differentially expressed gene; RPKM, reads per kilobase per million reads.

(GO 0005488; 75.60\%) and catalytic activity (GO 0008152; $36.40 \%)$. The majority of the biological process categories were associated with metabolic processes (GO 0008152; 49.70\%) and cellular processes (GO 0009987; 67.50\%) (Fig. 4).

The KEGG pathway database was used to perform the pathway analysis to understand further the biological function of the gene products. KOBAS was used to perform the KEGG pathway enrichment analysis. A KEGG analysis identifies the molecular interaction networks that are specific to particular organisms in cells with variants. It was identified that the mitogen-activated protein kinase (MAPK) signaling pathway (32 DEGs; 4.87\%), the DNA replication (15 DEGs; 2.28\%), the Toll-like receptor signaling pathway (19 DEGs; $2.89 \%$ ), the regulation of actin cytoskeleton (38 DEGs; 5.78\%), the metabolic pathways (91 DEGs; 13.85\%), the cytokine-cytokine receptor interaction (23 DEGs; 3.5\%), the phagosome (23 DEGs; 3.5\%), the focal adhesion (25 DEGs; 3.81\%), the chemokine signaling pathway (17 DEGs; $2.59 \%$ ), the natural killer cell-mediated cytotoxicity (10 DEGs; $1.52 \%$ ), the vascular endothelial growth factor (VEGF) signaling pathway (10 DEGs; 1.52\%) and 


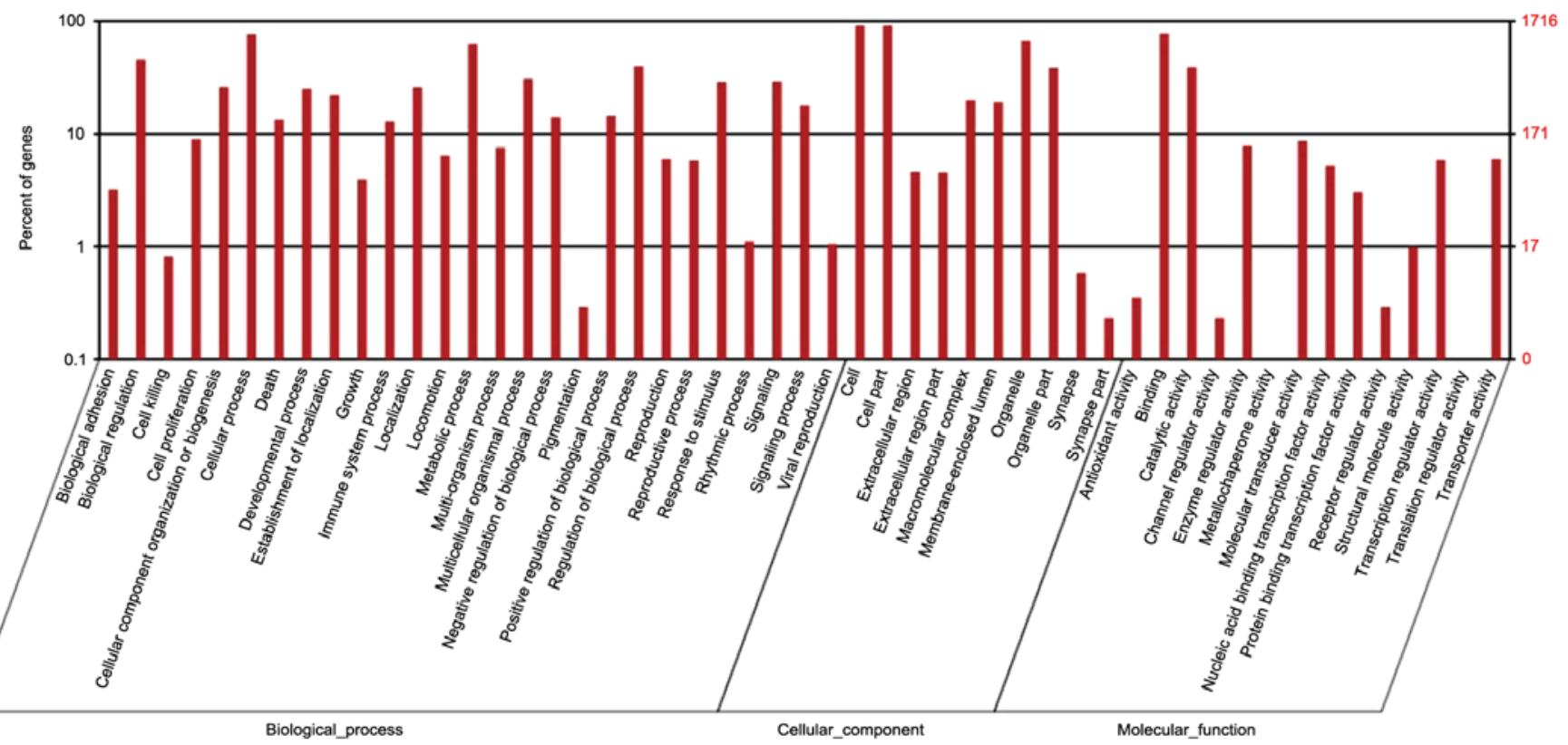

Figure 4. Gene Ontology functional classification of the differentially expressed genes.
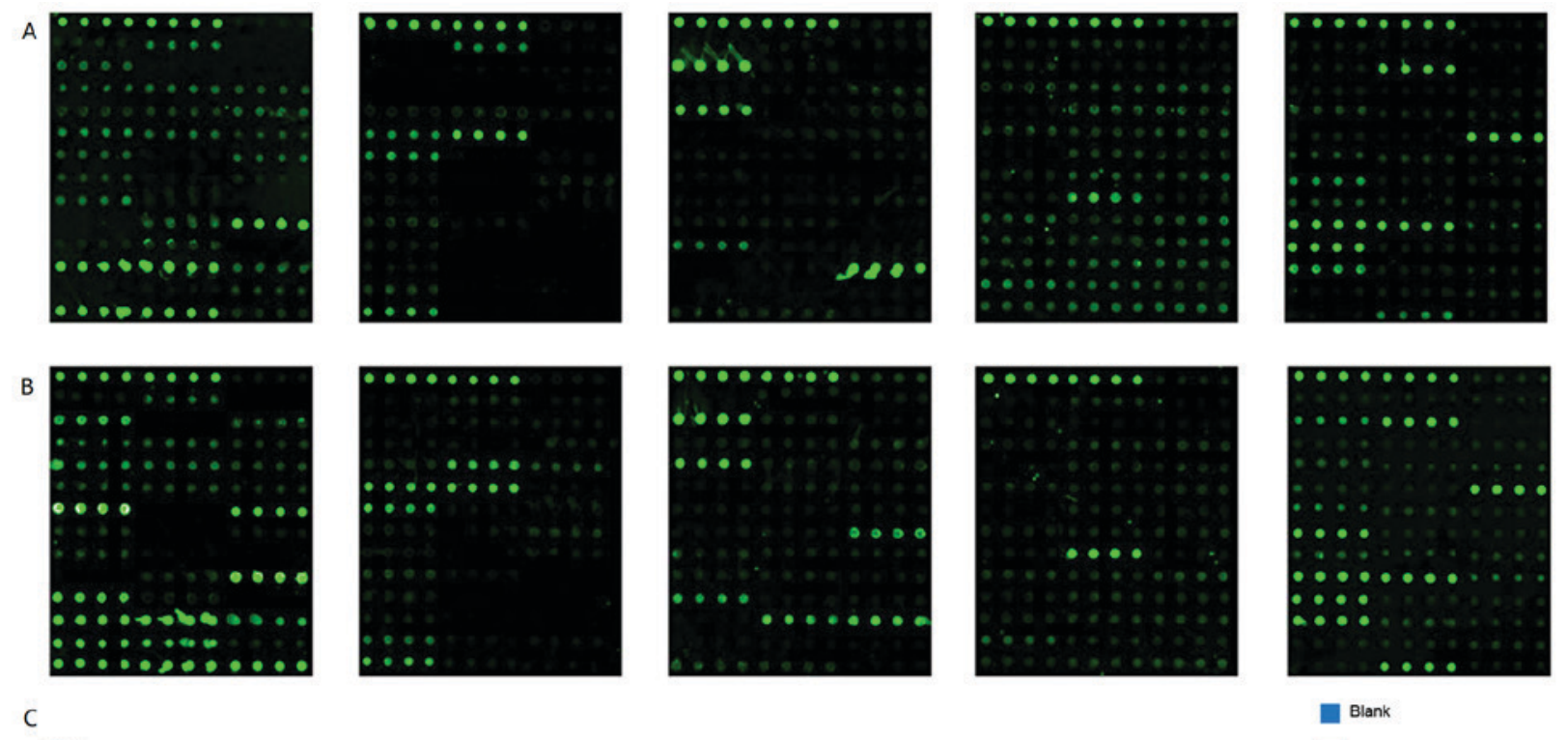

Blank

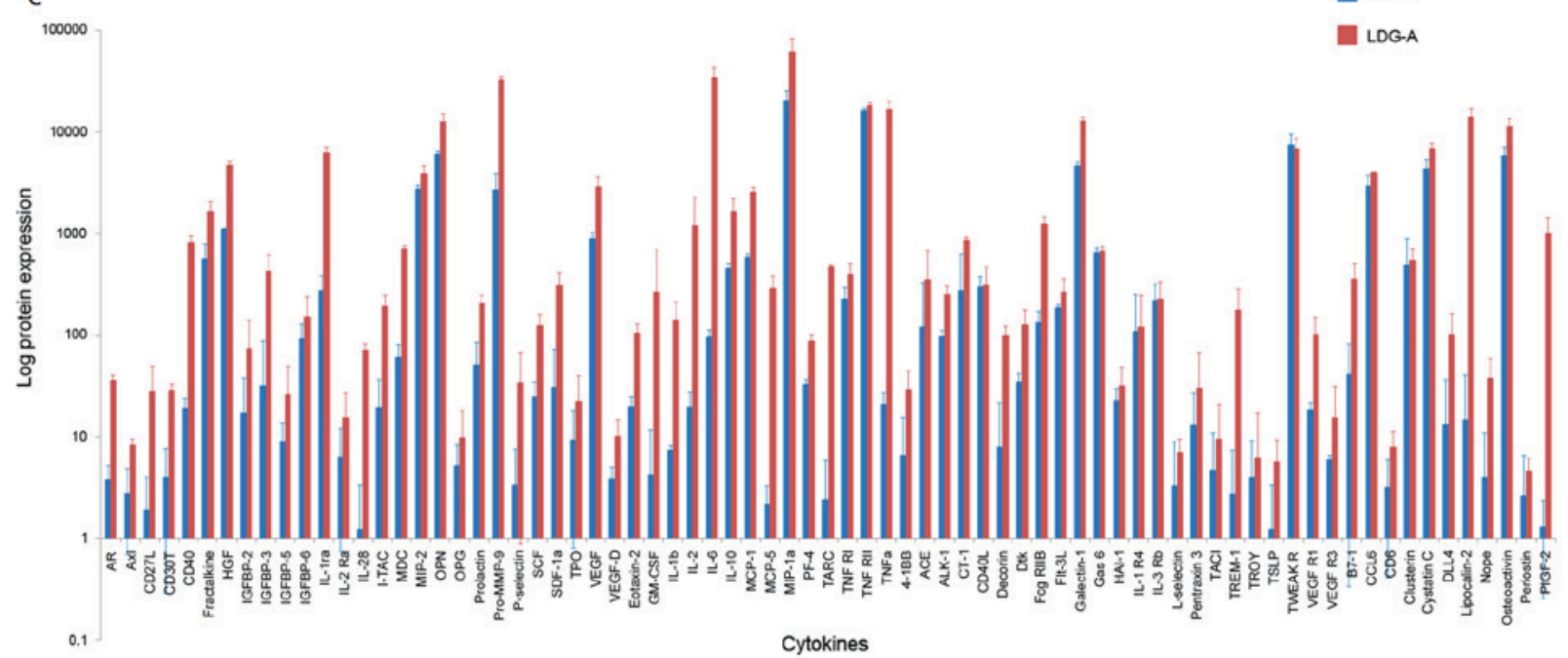

Figure 5. (A) Analysis of the protein microarray assay of the LDG-A group. (B) Analysis of the protein microarray assay of the blank control group. (C) Quantification of cytokine protein expression levels. 


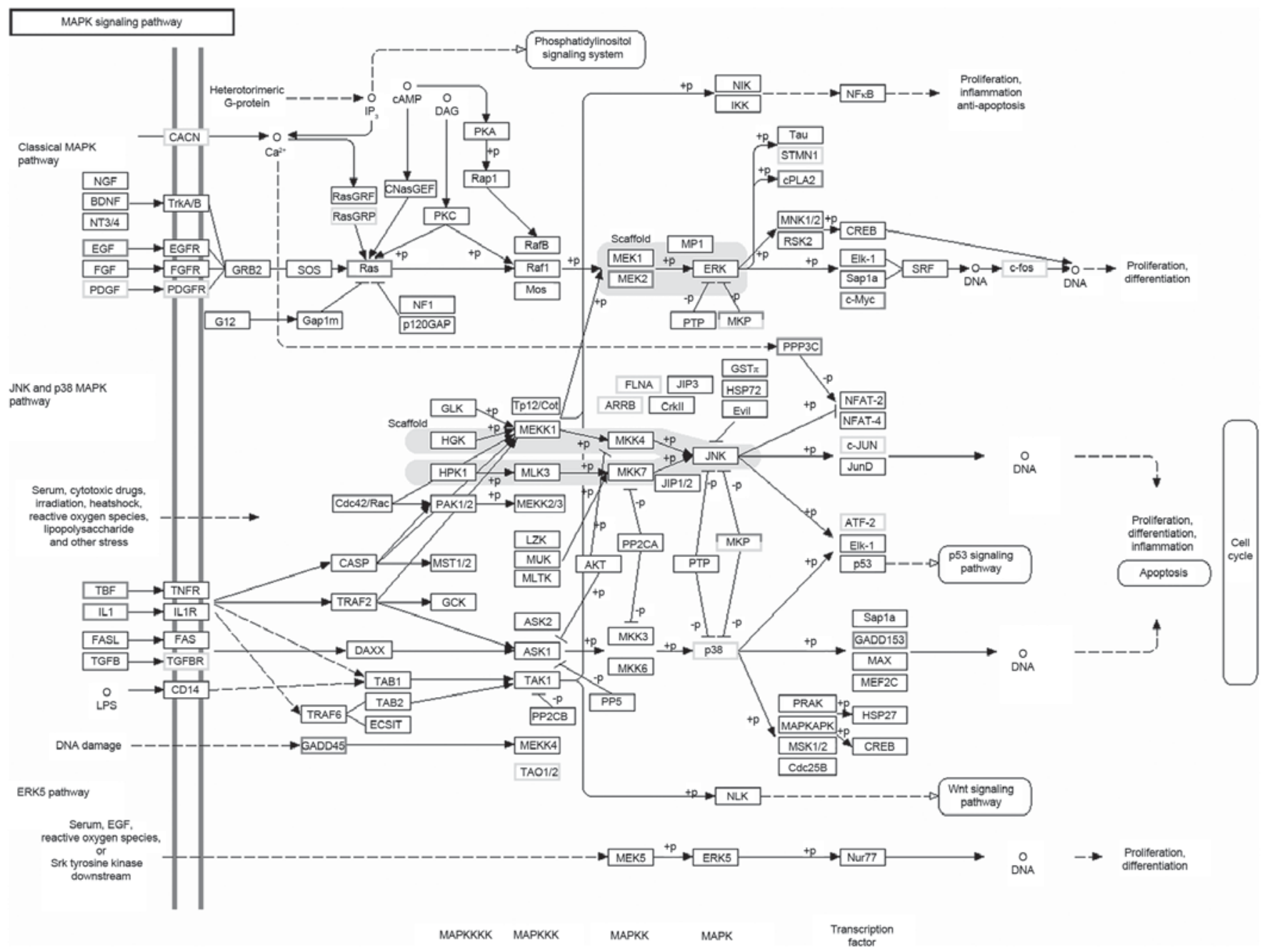

Figure 6. Mitogen-activated protein kinase signaling pathway. Reproduced from with permission under the terms of the Creative Commons Attribution License (CC-BY).

the transforming growth factor (TGF)- $\beta$ signaling pathway (10 DEGs; $1.52 \%$ ) were significantly enriched in the DEGs between the two cell groups. It is worth noting that the cell cycle between the two cell groups (27 DEGs with pathway annotation; $4.11 \%$ ) is also significantly enriched for DEGs. These results are consistent with the hypothesis that LDG-A could promote the proliferation of macrophages by promoting cell cycle progression in $\mathrm{G}_{0} / \mathrm{G}_{1}$ phase and eliminating cell cycle arrest in $\mathrm{G}_{2} / \mathrm{M}$ phase, which may induce cell division.

Changes in cytokines secreted by macrophages. The protein microarray experiments identified 200 cytokines (Fig. 5), which enabled the identification of 76 upregulated cytokines, including androgen receptor, cluster of differentiation (CD)27 ligand, CD30T, CD40 and fractalkine, and 44 downregulated cytokines, including CXC chemokine ligand 16, epidermal growth factor (EGF), insulin-like growth factor (IGF)-1, interleukin (IL)-2 and IL-21, and 82 cytokines with no change, including E-selectin, IGF-binding protein 5, T cell activation 3, CD27, cytotoxic T lymphocyte antigen 4, Dickkopf 1 and matrix metalloproteinase-10 (Fig. 5). Using KEGG pathway analysis, polysaccharide LDG-A was identified to use a range of signaling pathways to induce the immune response of macrophages, including the JAK (Janus kinase)/STAT (signal transducer and activator of transcription) signaling pathway, the TNF (tumor necrosis factor) signaling pathway, the nuclear factor $\kappa \mathrm{B}$ signaling pathway, the phosphoinositide 3-kinase/protein kinase $\mathrm{B}$ signaling pathway, the $\mathrm{T}$ cell receptor signaling pathway, pathways in cancer, the TGF- $\beta$ signaling pathway and the MAPK signaling pathway (Fig. 6). These results are also consistent with the hypothesis that LDG-A could promote the proliferation of macrophage cells by promoting cell cycle progression in $\mathrm{G}_{0} / \mathrm{G}_{1}$ phase and eliminating cell cycle arrest in $\mathrm{G}_{2} / \mathrm{M}$ phases, which may induce cell division.

\section{Discussion}

LDG-A exhibits significant antitumor activities in vivo. However, the antitumor activities and mechanism of action of LDG-A remain unclear. In the present study, the cell cycle analysis of macrophages and B cells was performed, and the transcriptomes of LDG-A group macrophages and control group macrophages were sequenced to identify DEGs using Illumina sequencing technology and to determine the molecular mechanisms of the antitumor and immunomodulatory activities of LDG-A in macrophages. In addition, a 
protein chip assay was used to identify the cytokines secreted by macrophages. In conclusion, LDG-A could promote the proliferation of $\mathrm{B}$ cells by promoting cell cycle progression in $\mathrm{S}$ phase and $\mathrm{G}_{2} / \mathrm{M}$ phase and eliminating cell cycle arrest in $\mathrm{G}_{0} / \mathrm{G}_{1}$ phase, and could promote the proliferation of macrophages by promoting cell cycle progression in $\mathrm{G}_{0} / \mathrm{G}_{1}$ phase and eliminating cell cycle arrest in $\mathrm{G}_{2} / \mathrm{M}$ phase, which may induce division of these cells. The effect of LDG-A on cell cycle was similar to that of LPS, which is a mitogen that promotes cell division and proliferation. The RPKM analysis indicated that the RPKM of the Fth1 gene was 78,249 in the LDG-A group, but only 16,044 in the control group.

Ferritin is a major intracellular iron storage protein in eukaryotes. It is composed of 24 subunits of heavy and light ferritin chains. The Fth1 gene encodes the heavy subunit of ferritin. Variation in ferritin subunit composition may affect the rates of iron uptake and release in different tissues. A major function of ferritin is the storage of iron in a soluble and non-toxic state. Defects in ferritin proteins are associated with several neurodegenerative diseases. This gene has multiple pseudogenes, and several alternatively spliced transcript variants have been identified, but their biological validity has not been determined. Ferritin Heavy Subunit is also associated with macrophage aggregation and polarization $(18,19)$.

The GO enrichment analysis and the KEGG pathway enrichment analysis revealed that the JAK/STAT, MAPK, chemokine, VEGF and TGF- $\beta$ signaling pathway, among others, are significantly enriched for DEGs. In total, 32 DEGs were identified in the MAPK signaling pathway, 15 of which were upregulated in the LDG-A group, including TNF [K03156; 24835 (1.2)], EGF [K04357; 294559 (2.1)], IL-1 [K04383; 24493 (3.6) and K04519; 24494 (1.8)], Ras [K07829; 361568 (1.3)], MAPK/extracellular-signal-regulated kinase kinase (MEK) 2 [K04369; 58960 (1.1)], c-Myc [K04377; 24577 (3.7)], protein phosphatase 2B catalytic subunit [K04348; 171378 (1.2)], and growth arrest and DNA damage 153 [K04452; 29467 (2.9)], whereas 17 were downregulated, including c-fos (K12362; 313874 (-2.3)] and c-Jun [K03283; 24468 (-1.5)]. The basic component of the MAPK signaling pathway is a conserved three-stage kinase pattern from yeast to humans, and is one of the most important pathways in eukaryotic signaling networks and serves a key function in the regulation of gene expression and cytoplasmic function, including MAPK, MEK (also known as MAPK kinase) and MEK kinase (also known as MAPK kinase kinase). These three kinases may be activated sequentially and regulate cell proliferation, differentiation, stress adaptation to the environment, etc. (20). EGF is a growth factor in the MAPK signaling pathway. Its principal function is to promote the division of cells. Studies have indicated that a small amount of EGF can strongly stimulate cell proliferation, inhibit the expression of aging genes, prevent cell aging and maintain the best physiological state of cell components $(21,22)$. This process would adequately explain the mechanism of LDG-A's proliferative activity on macrophages. The results of the present study provide a good foundation for further studies.

\section{Acknowledgements}

Not applicable.

\section{Funding}

This project was supported by the Science and Technology Support Project of Sichuan Province (grant nos. 2018JY0087 and 2018NZ0055), the Cultivate Major Projects of Sichuan Province (grant no. 16CZ0018), Nanchong Science and Technology Bureau of Sichuan Province (grant no. 16YFZJ0043), Talent Program of China West Normal University (grant nos. 17YC328, 17 YC136 and 17YC329), National Training Project of China West Normal University (grant no. 17c039) and Innovative Team Project of China West Normal University (grant no. CXTD2017-3).

\section{Availability of data and materials}

The datasets used and/or analyzed during the current study are available from the corresponding author on reasonable request.

\section{Authors' contributions}

YH conceived the presented idea and carried out the experiment. $\mathrm{YH}$ and $\mathrm{MW}$ analyzed the data and wrote the manuscript. DZ, LL, XD and WH interpreted the data, discussed the results and implications, commented on the manuscript at all stages and revised the manuscript critically. All authors gave final approval of the version to be published and agreed to be accountable for all aspects of the study in ensuring that questions related to the accuracy or integrity of any part of the study are appropriately investigated and resolved.

\section{Ethics approval and consent to participate}

Not applicable.

\section{Patient consent for publication}

Not applicable.

\section{Competing interests}

The authors declare that they have no competing interests.

\section{References}

1. Song G and Du Q: Structure characterization and antitumor activity of an $\alpha \beta$-glucan polysaccharide from auricularia polytricha. Food Res Int 45: 381-387, 2012.

2. Schepetkin I and Quinn M: Botanical polysaccharides: Macrophage immunomodulation and therapeutic potential. Int Immunopharmacol 6: 317-333, 2006.

3. Zhao Z, Li J, Wu X, Dai H, Gao X and Liu M: Structures and immunological activities of two pectic polysaccharides from the fruits of Ziziphus jujuba Mill. cv. jinsixiaozao Hort. Food Res Int 39: 917-923, 2006.

4. Lee K and Jeon Y: Macrophage activation by polysaccharide isolated from astragalus membranaceus. Int Immunopharmacol 5: 1225-1233, 2005

5. Song JY, Han SK, Son EH, Pyo SN, Yun YS and Yi SY: Induction of secretory and tumoricidal activities in peritoneal macrophages by ginsan. Int Immunopharmacol 2: 857-865, 2002.

6. Ma H, Liu G, Ding W, Wu Y, Cai L and Zhao Y: Diabetes-induced alteration of F4/80+ macrophages: A study in mice with streptozotocin-induced diabetes for a long term. J Mol Med (Berl) 86: 391-400, 2008 
7. Di Carlo E, Forni G, Lollini P, Colombo M, Modesti A and Musiani P: The intriguing role of polymorphonuclear neutrophils in antitumor reactions. Blood 97: 339-345, 2001

8. Chen $\mathrm{G}$ and Goeddel D: TNF-R1 signaling: A beautiful pathway. Science 296: 1634-1635, 2002.

9. Cheng A, Wan F, Wang J, Jin Z and Xu X: Macrophage immunomodulatory activity of polysaccharides isolated from Glycyrrhiza uralensis fish. Int Immunopharmacol 8: 43-50, 2008.

10. Chihara G, Maeda Y, Hamuro J, Sasaki T and Fukuoka F: Inhibition of mouse sarcoma 180 by polysaccharides from Lentinus edodes (Berk.) sing. Nature 222: 687-688, 1969.

11. Kobayashi H, Yoshida R, Kanada Y, Fukuda Y, Yagyu T, Inagaki K, Kondo T, Kurita N, Suzuki $\mathrm{M}$ and Terao T: Suppressing effects of daily oral supplementation of beta-glucan extracted from agaricus blazei Murill on spontaneous and peritoneal disseminated metastasis in mouse model. J Cancer Res Clin Oncol 131: 527-538, 2005.

12. Nakazato H, Koike A, Saji S, Ogawa N and Sakamoto J: Efficacy of immunochemotherapy as adjuvant treatment after curative resection of gastric cancer. Study Group of Immunochemotherapy with PSK for gastric cancer. Lancet 343: 1122-1126, 1994.

13. Shin JY, Song JY, Yun YS, Yang HO, Rhee DK and Pyo S: Immunostimulating effects of acidic polysaccharides extract of panax ginseng on macrophage function. Immunopharmacol Immunotoxicol 24: 469-482, 2002.

14. Ding X, Hou Y and Hou W: Structure feature and antitumor activity of a novel polysaccharide isolated from Lactarius deliciosus Gray. Carbohydr Polym 89: 397-402, 2012.

15. Hou Y, Ding X, Hou W, Song B, Wang T, Wang F and Zhong J: Immunostimulant activity of a novel polysaccharide isolated from Lactarius deliciosus (L. ex Fr.) gray. Indian J Pharm Sci 75: 393-399, 2013
16. Jianwei G, Jianzhong L, Bin L and Zhensheng L: Isolation and purification of functional total RNA from blue-grained wheat endosperm tissues containing high levels of starches and flavonoids. Plant Mol Biol Rep 19: 185-186, 2001.

17. Wagner GP, Kin K and Lynch VJ: Measurement of mRNA abundance using RNA-seq data: RPKM measure is inconsistent among samples. Theor Biosc 131: 281-285, 2012.

18. Zhang Y, Meng Q, Jiang T, Wang H, Xie L and Zhang R: A novel ferritin subunit involved in shell formation from the pearl oyster (Pinctada fucata). Comp Biochem Physiol B Biochem Mol Biol 135: 43-54, 2003.

19. Honarmand Ebrahimi K, Bill E, Hagedoorn P and Hagen W: The catalytic center of ferritin regulates iron storage via $\mathrm{Fe}(\mathrm{II})-\mathrm{Fe}(\mathrm{III})$ displacement. Nat Chem Biol 8: 941-948, 2012.

20. Orton R, Sturm O, Vyshemirsky V, Calder M, Gilbert D and Kolch W: Computational modelling of the receptor-tyrosine-kinase-activated MAPK pathway. Biochem J 392: 249-261, 2005.

21. Herbst R: Review of epidermal growth factor receptor biology. Int J Radiat Oncol Biol Phys 59 (Suppl 2): S21-S26, 2004.

22. Nagashima T, Suzuki T, Kondo S, Kuroki Y, Takahashi K, Ide K, Yumoto N, Hasegawa A, Toyoda T, Kojima T, et al: Integrative genome-wide expression analysis bears evidence of estrogen receptor-independent transcription in heregulin-stimulated MCF-7 cells. PLoS One 3: e1803, 2008. 